\title{
Smart Light Stick: An Interactive System for Pop Concert
}

\author{
Jing Yang ${ }^{1, a}$, Yue Bai ${ }^{2, b}$ and Jundong Cho ${ }^{3,4, c, *}$ \\ ${ }^{1}$ College of Information and Communication Engineering, Sungkyunkwan University, Seobu Road, Suwon, \\ South Korea \\ ${ }^{2}$ School of Electronics and Information, Northwestern Polytechnical University, West Youyi Road, Xi'an, \\ China \\ ${ }^{3}$ Department of Electrical and Electronic Engineering, Sungkyunkwan University, Seobu Road, Suwon, \\ South Korea \\ ${ }^{4}$ National Key Laboratory for Electronic Measurement Technology, North University of China, Xueyuan \\ Road, Taiyuan, China \\ a yangjing24@skku.edu, bythemailforyou@gmail.com, ${ }^{\mathrm{c}}$ jdcho@skku.edu \\ *corresponding author
}

Keywords: pop concert, interaction, audience participation.

\begin{abstract}
People's living standards have improved with the development of society, and concerts have already become part of our daily lives. Though there are thousands of pop concerts around the world each year, the interaction between idol and fan during the show is still primitive and limited, and almost every stage of the concert is controlled by the host and the performer. These obstacles have a profound effect on the audience's experience and become a bottleneck in the popularization of advanced pop culture. In this work, we present a smart interactive system for pop concerts, which helps to facilitate communication between participants. In this study, we propose a new type of light bar to encourage the active participation of the audience. We conducted an evaluation with 10 participants and found that this system would enhance the audience experience and stimulate dialogue.
\end{abstract}

\section{Introduction}

Pop concert refers to concert of popular and light classical music played by a symphony orchestra [1]. Recently, it has gained popularity as a storm. There are three main types of pop music: K-POP, J-POP and Western POP. Despite the popularity of pop music, the interactions are minimal during the show. In current pop concert, almost everything are controlled by the organizers and performers, spectators can do nothing but cheer strenuously for their favorite idol. Even though they shout at the top of their voice, idols never know their effort, and thus their enthusiasm can be discouraged.

Interaction within the context of musical performance has been subject to a lot of research over the last decades. For instance, there already exist sensor-based systems allow for the perception of bodily interaction (e.g. [2][3]) and tangibles to enable intuitive and appropriate interaction with digital devices (e.g. [4][5]). Also, there are network-based systems that make collaborative performances possible where participants are getting closer to each other even though in some cases they are spatially divided (e.g. [6][7][8]) [9].

The original inspiration for our work was stemmed from the Be a Judge system [10]. This system, which was presented at CHI 2004 Student Competition, utilizes wireless motion sensors and microphones to cast spectators' vote in real time during the Olympic Games. When viewers watch sports games, they use natural actions such as applause and cheering to vote. However, we believe that the usage of loudness can be misled by booing and noise. The system also has limitations that can not be used consistently because some players appear at the same time during some sporting events such as gymnastics. 
In this paper, we raised an interactive system that spectators have to shake a redesigned light stick to blend into the pop concert. The reason why we decided to integrate interactive elements into a light stick is because it is widely used in current pop concerts. For example, almost every idol group in South Korea released their official color and stick (see Figure 1). So it is usual to see fans cheer for their favorite idols during the concert by using the official stick.

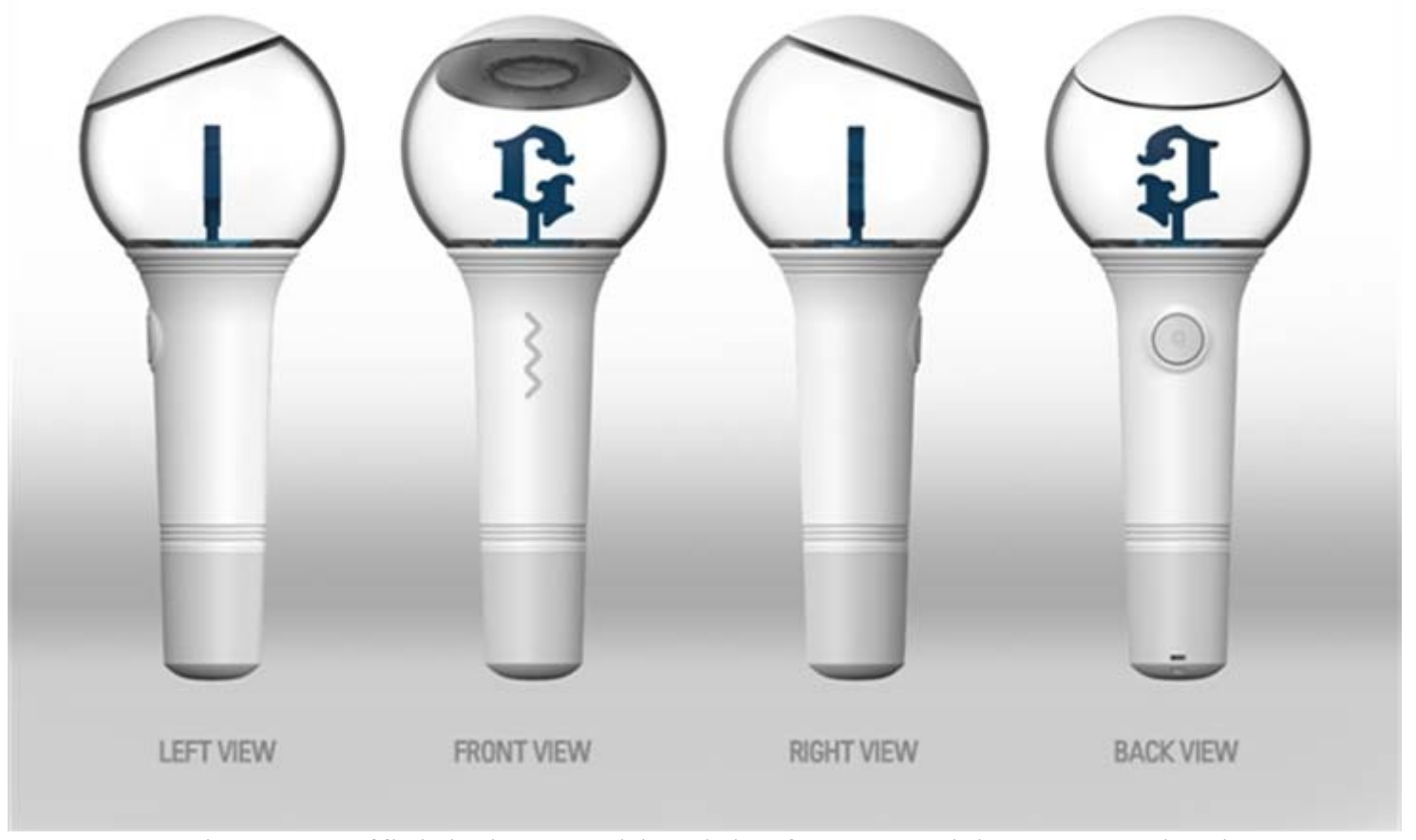

Figure 1. Official glass marble stick of Korean girl group GFriend

This system makes three important contributions to concert interaction. First, it makes spectators become a part of the concert. Second, we add social part to our system, spectators can chat and share their concert experience with strangers during or even after the concert. Third, spectators who watch the concert by TV or computer can also join the real-time interaction.

\section{Preliminary Survey}

Before the development, we conducted a questionnaire survey among 34 senior pop fans. The objective is to know their opinions about the level of interaction in current pop concert and expectation of new interactive system. According to our finding, $53 \%$ of the participants are not satisfied with the interaction during current concert and $94 \%$ of them think it is necessary to develop an interactive system to improve the present situation. 25 of the participants consider this kind of device should be provided by the organizers, thus the light stick is a good choice since it is very cheap and easy production. 22 of the participants usually watch pop concerts through webcast and almost all fans hope to engage in the concert interaction even though they are not in the scene. In this process, we received positive feedback on our concept continuously.

\section{System Design}

In this section, we provided an overview of the workflow of the proposed system and described each module in detail.

Overview of system workflow

The overview of system workflow is depicted as in Figure 2. Firstly, user has to turn on the light stick and connect it with his or her smartphone by bluetooth. Once user begin to shake the light stick back and forth, the color of LEDs inside it will change according to the shake frequency (Sequence: blue - green - yellow - red), so that idols can know where are their fans and adjust their performance according to the real-time feedback. Secondly, the number of times that user shook 
will be sent to user's smartphone and then transmit to our web database. Thirdly, users who shake the light stick at the same or similar frequency at the same time are judged to have the same level of enthusiasm and can add friends through the mobile application. You can then chat with each other and share your personal experiences.

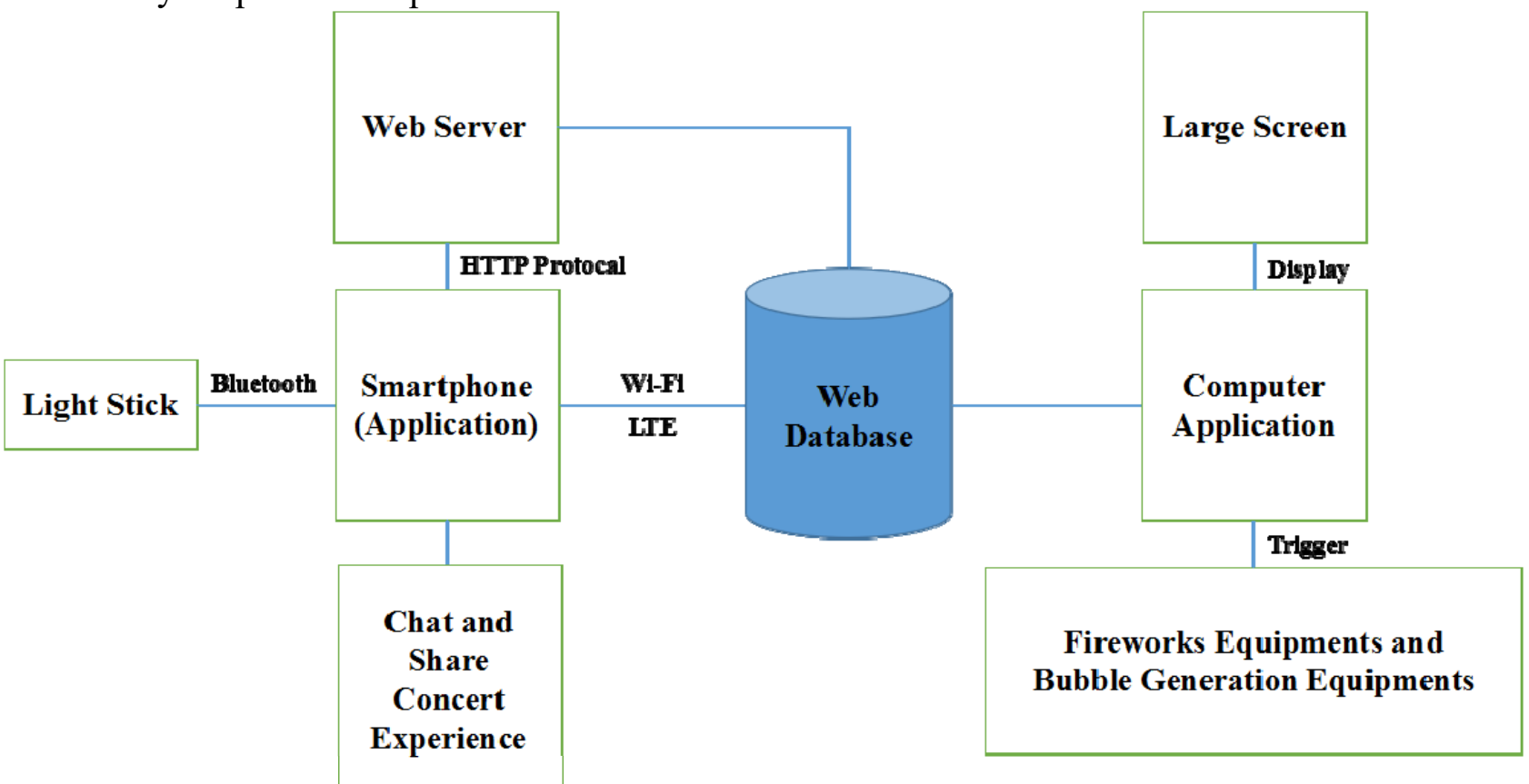

\section{Light stick}

Figure 2. Overview of system workflow

The light stick is made from a common plastic stick (see Figure 3), which integrates an Arduino Mini, a triaxial accelerometer, an HC-05 bluetooth module, some LEDs and a rechargeable battery. Once users turn on and shake the light stick correctly, the triaxial accelerometer inside it will work and detect the shake frequency. The Arduino then controls the color of the LEDs according to the vibration frequency and calculates the number of times the user shakes. Finally, these two values are sent via bluetooth to your smartphone.

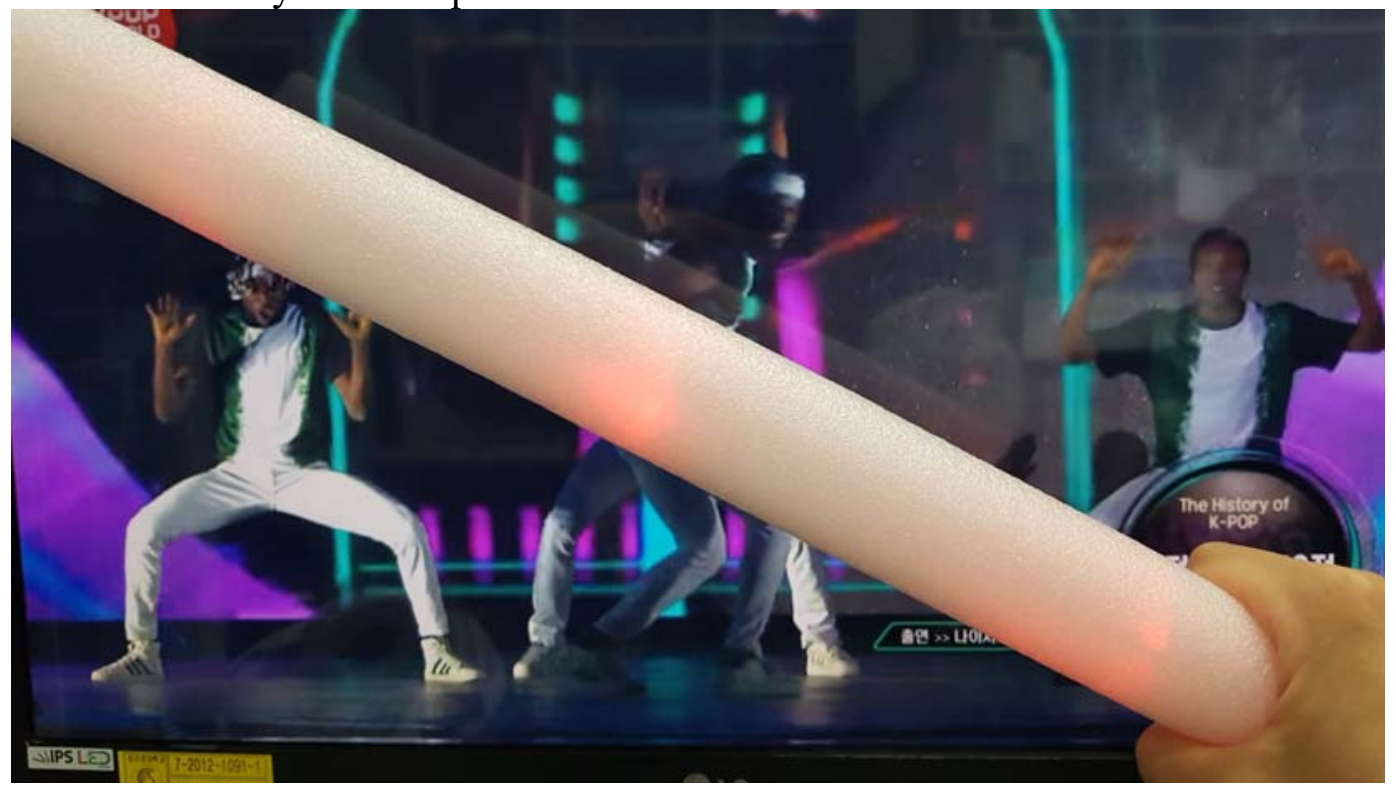

Figure 3. Light Stick

\section{Mobile application}

The mobile application has been developed based on Android Operating System (see Figure 4) and we will release the iOS version in the future. Users can see the number of shakes in real time and all values received from the light stick are sent to the web database. At the same time, the target value and current total value (sum of all users in real time) can also be checked so that users can 
know how much more contribution they should make. Fans who shake at the same frequency at the same time seem to be fans of the same idol or idol group, so they can add friends and share their concert experience with each other through this application.

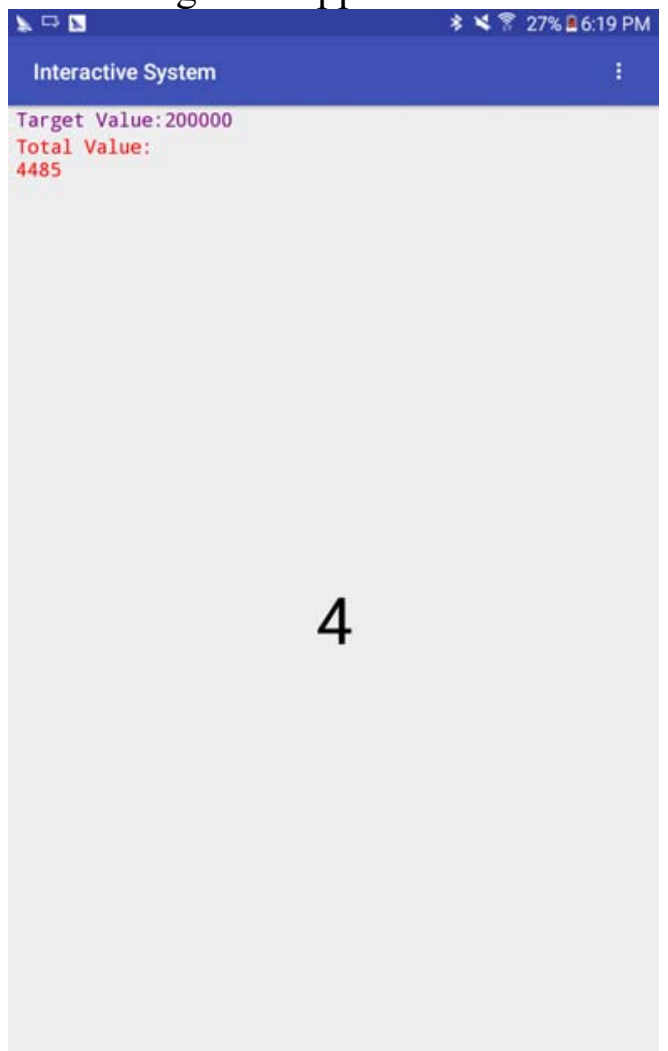

Web database

Figure 4. Mobile application

The web database is used to store user information and the temporary values.

Computer application

The computer application can get current total value from the database and show it in the large screen. The goal value set by the organizer is also displayed. When the total value reaches the target value, the fireworks equipment and the bubble generator will operate. Abovementioned values will be reset after the performance of each performer. Also, the target value should be set manually since the number of fans of different idol or idol group is different.

Web server

We built a web server to achieve chatting and sharing functions. The web server exchanges message with the application by HTTP protocol.

\section{Initial Evaluation}

The aim of the initial evaluation was to get insight into the experience of the audience when watching musical performances with our system. Because of the limitation of conditions, we cannot conduct a formal evaluation in real pop concert so far.

\section{Setting}

The performance was held in a classroom. We invited a student band to perform on the platform and we used the projector to simulate the large screen. We asked participants to sit in the center of the classroom which allowed all members of the audience to observe the performer's action and the projector properly.

\section{Audience}

The audience was 10 persons between 20 and 35 years old (50\% female). All of them had previously attended at least one pop concert.

Procedure

First, we explained the aim of this evaluation and asked all participants to install our mobile 
application. Second, the student band began to perform and audiences were asked to shake the light stick and checked the values through the application. Third, participants were given a questionnaire with 12 questions about their experiences and opinions to our system. In all, the evaluation lasted about 30 minutes.

\section{Results}

According to the questionnaires and our observation, we found that the interaction was improved during the performance, participants were willing to communicate with people who sat near from them and ask others to shake the light stick to support their favorite idols. Most participants were satisfied with this new system and almost all of them wrote that it was a big improvement since they can join the concert even though they are not on the spot by using our light stick and mobile application. Several people wrote that the social part was amazing, because they can make friends with strangers who has the same hobby. However, several fans were worried about the issue that once they focus on the interaction they will not pay attention to the performance.

\section{Conclusion}

Audience participation is crucial for musical performance. However, current pop concerts lack of interaction and social communication. In this work, we have presented an innovative solution to improve the interaction and spectator experience during the pop concert and stimulate conversation.

In the future, we would like to conduct a formal evaluation of our interactive system in an actual pop concert and improve the system according to the feedback.

\section{Acknowledgements}

This research was supported by the Ministry of Trade, Industry and Energy (MOTIE), Korea, through the Education Support program for Creative and Industrial Convergence (Grant Number S2016-0117-000).

\section{References}

[1] "pop concert". Dictionary.com Unabridged. Random House, Inc. 18 Aug. 2017. $<$ Dictionary.com http://www.dictionary.com/browse/pop-concert>.

[2] M. Feldmeier and J. A. Paradiso, "An Interactive Music Environment for Large Groups with Giveaway Wireless Motion Sensors,” Computer Music Journal, vol. 31, no. 1, pp. 50-67, 2007.

[3] R. Knapp and B. Bortz, MobileMuse: Integral Music Control Goes Mobile, Proc NIME, 2001.

[4] E. W. Pedersen and K. Hornbæk, “mixiTUI,” pp. 223-230, 2009.

[5] M. Kaltenbrunner, G. Geiger, and S. Jordà, Dynamic patches for live musical performance, pp. 19-22, 2004.

[6] G. McAllister and M. Alcorn, "Interactive performance with wireless PDAs," Proc ICMC, 2004.

[7] J. Young, "Using the Web for live interactive music," pp. 302-305, 2001.

[8] M. Rohs and G. Essl, CaMus2: Collaborative Music Performance with Mobile Camera Phones, Proc ACE, pp. 190-195, 2007.

[9] Oliver Hödl, Fares Kayali, Geraldine Fitzpatrick, DESIGING INTERACTIVE AUDIENCE PARTICIPATION USING SMART PHONES IN A MUSICAL PERFORMANCE, ICMC 2012, September 9-14, 2012, Ljubljana, Slovenia.

[10] W. Aigner, M. Tomitsch, M. Stroe and R. Rzepa, Be a Judge! - Wearable Wireless Motion Sensors for Audience Participation, CHI 2004, April 24-29, 2004, Vienna, Austria. 